\title{
Biofilm Formation of Agrobacterium tumefaciens on Abiotic Surface is Independent of Cellulose Synthesizing Gene
}

\author{
Reshma Tuladhar \\ Central Department of Microbiology, Tribhuvan University, Kathmandu, Nepal \\ e-mail: resutu@gmail.com
}

\begin{abstract}
Surface attachment is indispensable in the process of biofilm formation in Agrobacterium tumefaciens, which has been attributed to the production of extracellular polysaccharide. Adherence of bacteria on the plant was expected to be strong with cellulose synthesis. However, its role in the biofilm formation on abiotic surface is not known. Biofilm cover-slip assay was carried to estimate the biofilm formation. Biofilm formation was not reduced by truncating cellulose synthesis gene. The celCD deleted strain produced biofilm slightly greater than normal strain NTL4 of A. tumefaciens. Besides, production of extracellular polysaccharide was not reduced in the cellulose gene deleted strain NTL4 $\Delta$ celCD. Quantitative estimation showed increased exo-polysaccharide in celCD deleted strain. Abolishing the cellulose synthesis property by truncating the cellulose gene did not affect biofilm formation in abiotic surface. In fact cellulose might be repressing biosynthesis of other polysaccharides responsible in biofilm formation.
\end{abstract}

Key words: biofilm cover-slip assay, exo-polysaccharide, calcofluor assay

\section{Introduction}

Agrobacterium tumerfaciens is a gram-negative soil bacterium that forms crown gall tumors in most dicotyledonous plants. Crown gall formation is the result of the infections of the wound sites in the plant (Reuhs et al. 1997). Pathogenesis involves the horizontal transmission of a segment of A. tumefaciens DNA carried on the tumor-inducing (Ti) plasmid into the host plant genome (Gelvin 2003). Genes on the transferred DNA direct a hormonal imbalance in the plant that results in uncontrolled proliferation of plant cells forming the characteristic gall. An early step in tumor formation is the attachment of the bacteria on the plant cell surface (Matthysse 1986).Surface attachment is indispensable in the process of the host plant infection and biofilm formation in $A$. tumerfaciens.

Microbial biofilms are defined as surface-associated, multicellular assemblages of organisms (Parsek \& Fuqua 2004). Organic compounds released by plant serve as powerful attractant for A. tumefaciens (Binns et al. 1992), which initiates the attachment. Formation of biofilm begins with the attachment of individual or small groups of bacteria to the surface. This is followed by bacterial growth and additional attachments to form a biofilm. Large amounts of extracellular matrix materials are often produced during biofilm formation (Matthysse et al. 2005). This matrix holds the cells in association with each other and the surface and commonly contains exopolysaccharides (EPS) (Sutherland 2001).

An EPS produced by $A$. tumefaciens and closely related Rhizobium meliloti is succinoglycan, which consists of repeating units of linked glucose and galactose with acidic sidegroups. Mutants of $R$. meliloti, which lack this EPS induced ineffective nodules on alfalfa (Cangelosi et al. 1987). Besides, several other bacteria are known to produce polysaccharides contributing to the formation of biofilm. Overproduction of Vibrio polysaccharide in 
Vibrio cholerae has been associated with increased biofilm formation and elevated synthesis of EPS in opportunistic human pathogen Pseudomonas aeruginosa form dense biofilms on inert surfaces as well as human tissue (Mathee et al. 1999, Matthysse et al. 2005). However, overproduction of EPS does not result in a hyperadherent phenotype in all biofilm forming bacteria (Parsek \& Fuqua 2004).

Cellulose is frequently found component of the biofilm matrix in many organisms including members of the Rhizobiaceae (Flemming \& Wingender 2010, Bogino et al. 2013). A. tumefaciens in presence of cellulose fibrils bind tightly to the plant surface while the mutants show the reduced ability to colonize plants and induce tumor formation (Matthysse 1983). Over production of cellulose enhances attachment to plant roots in $A$. tumefaciens (Matthysse et al. 2005). Cellulose synthesis in A. tumefaciens requires genes in two operons, celABCG and celDE (Matthysse 1995, Matthysse et al. 2005). It is not confirmed that cellulose overproduction has any effect on biofilm formation on inert material. Thus, this work was carried out to study the effect of cellulose on the biofilm formation on inert material by A. tumefaciens.

\section{Methodology \\ Strain and growth condition}

The bacterial strains used were i) C58 (Wild type $A$. tumefaciens which extensively forms biofilm), ii) NTL4 (derivative of C58 with deletion in et $_{C 58}$ locus in plasmid pTi C58), iii) NTL4 celCD (derived from NTL4 with deletion in celCD locus of cellulose biosynthesis gene), iv) exoR (positive control for exopolysaccharide producer and v) exoA (negative control for exopolysaccharide producer). C58 (Wild type), NTL4 (pTi C58) and NTL4 $\Delta$ celCD were generously provided by Farrand SK, University of Illinois, IL. Bacteria were maintained on AT minimal medium supplemented with $0.5 \%$ (wt/vol) glucose and $15 \mathrm{mM}$ ammonium sulfate (ATGN).

\section{Analysis of static culture biofilms}

Polyvinyl chloride (PVC) coverslips were placed vertically in 12-well polystyrene cell culture plates (Corning Inc.) inoculated with cells in ATGN at an $\mathrm{OD}_{600}$ of 0.05 and incubated at room temperature for 48 hours. Biofilms were visualized macroscopically by staining with $1 \%$ Crystal Violet solution. For assessing biofilm by visualizing Crystal Violet, coverslips were rinsed in double-distilled water, stained with $0.1 \%$ (wt/vol) $\mathrm{CV}$, and rinsed again in double-distilled water. Biomass adhered to the coverslip was quantified by soaking stained coverslips in $1 \mathrm{ml}$ dimethyl sulfoxide (DMSO) to solubilize the $\mathrm{CV}$ and measuring the absorbance at $600 \mathrm{~nm}\left(A_{600}\right)$ in a Bio-Tek synergy HT microplate reader. Absorbance values were normalized to culture growth by dividing the $A_{600}$ value for solublized CV by the $\mathrm{OD}_{600}$ of the planktonic cells. Each of the experiment was carried out in triplicate.

\section{Calcofluor assay}

A. tumerfaciens strains were streaked onto ATGN agar containing $0.02 \%$ calcofluor (fluorescent brightener 28; Sigma F-3397) and incubated for growth. To determine if a strain produced exopolysaccharide, agar plates were exposed to long-wavelength UV light, and the bacterial streaks on the agar plates were monitored for bright blue fluorescence.

\section{Estimation of exopolysaccharide}

Anthrone method for estimation of glycosidic linked polysaccharide was performed for the estimation of exopolysaccharide. The bacterial cells were grown in ATGN at an $\mathrm{OD}_{600}$ of 0.08 . Four $\mathrm{ml}$ of the anthrone reagent was added to $1 \mathrm{ml}$ of the supernatant of cell culture.

\section{Results and Discussion Biofilm formation}

Wildtype and cellulose mutants were monitored by crystal violet staining for the attachment and static biofilm formation on PVC coverslips in ATGN. Visually the crystal violet stained in the biofilm formed by NTL4 parent strain which has intact cellulose biosynthesis gene and NTL4 $\Delta \quad$ celCD cellulose mutant were similar. The wildtype C58 form dense biofilms (Fig. 1). The CV staining was normalized for culture growth (CV absorbance divided by planktonic culture turbidity). The comparisons were made from the Crystal violet $\mathrm{A}_{600} / \mathrm{OD}_{600}$ data ( Fig. 2). The normalized data showed the overall biofilm formation capacity. The highest biofilm formation ability was found in the wildtype. The cellulose mutant NTL4 $\triangle \quad$ celCD showed a slight increase in biofilm formation compared to the the NTL4 strain. The deficiency in an intact cellulose biosynthesis gene did not affect the ability in the formation of biofilm. 

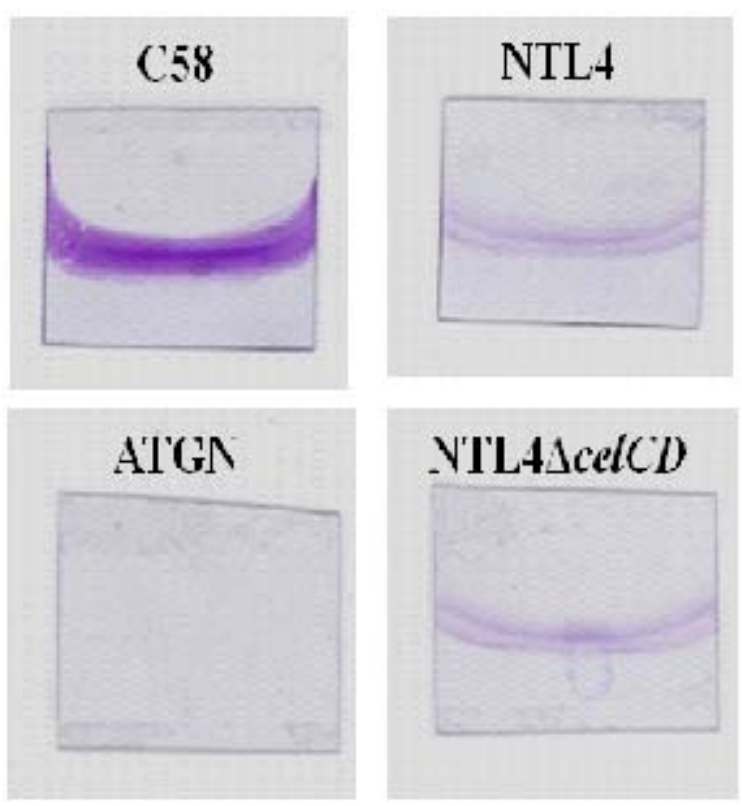

Fig. 1. Static biofilm formation and surface attachment (Crystal violet staining for the attachment on PVC coverslips in ATGN).

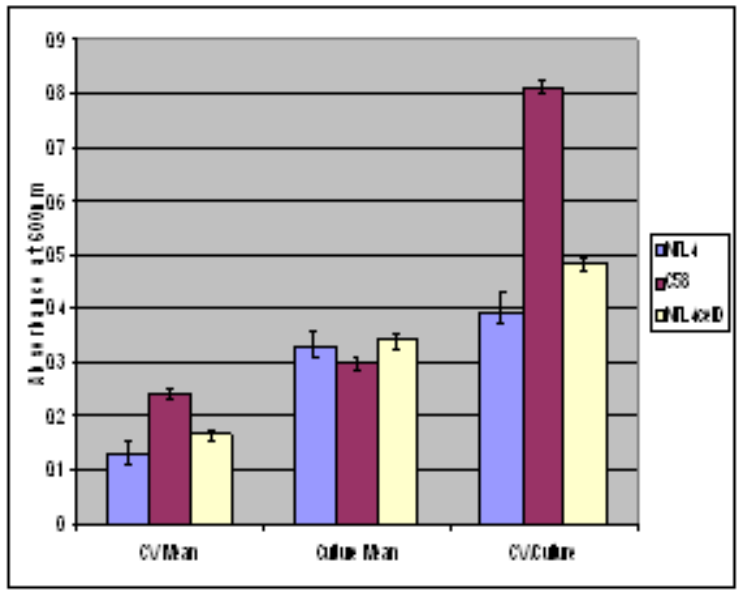

Fig. 2. Biofilm formation by different strains: Biofilms formed on PVC coverslip at 72 hpi were stained with $\mathrm{CV}$ and quantified from DMSO solubilized CV. CV mean (Adherent biomass $\mathrm{CVA}_{600}$ ), Culture mean (cell biomass OD600) and CV/Culture (biomass normalized for growth $\mathrm{CV} A_{600} / \mathrm{OD}_{600}$ ).

\section{Calcofluor assay for detection of exopoly- saccharide}

In order to assess the production of exopolysaccharide, the calcofluor assay was performed. Calcofluor binds polysaccharide, and the colonies producing polysaccharide emit bright blue fluorescence when they are exposed to longwavelength UV light. On ATGN agar containing 0.02\% calcofluor, the wild type C58, NTL4, NTL4 $\Delta$ cel CD and $e x o R$ (positive control for exopolysaccharide) emitted bright blue fluorescence when exposed to long-wavelength UV light. The negative control exoA showed the reduced fluorescence and almost appeared non fluorescent (Fig. 3). The cellulose deficient strain NTL4 $\Delta$ celCD showed fluorescence intensity identical to the w1ld type (C58) and positive control (exoR). This shows that polysaccharide other than cellulose is produced by cellulose deficient strain.

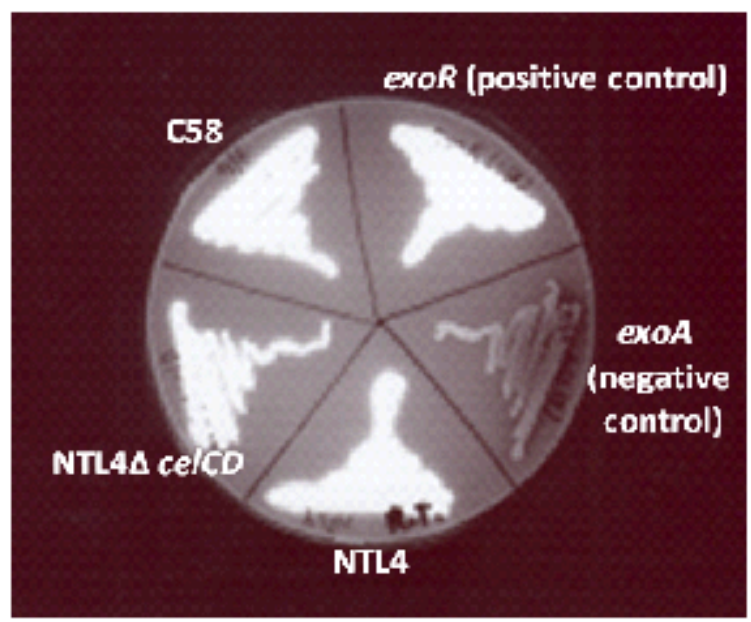

Fig. 3. Exopolysaccharide production in A. tumefaciens C58 (wt), NTL4, NTL4 $\Delta$ cel $C D$, exo $R$ and exoA. Strains were streaked on ATGN supplemnented with $0.02 \%$ calcofluor. The organisms were grown on plates and exposed to long-wavelength UV light. Bright fluorescent was observed in all the strains except for the negative control exoA.

\section{Estimation of expolysaccharide}

The exopolysaccharide produced by different strains was qualitatively estimated by the anthrone method. It was found that the cellulose mutant NTL4 $\Delta$ celCD produced nearly equal amount of polysaccharide as the wild type C58. However the polysaccharide concentration was lower in cellulose intact strain NTL4 from which the mutant was derived. Fairly small amount of carbohydrate was present in exopolysaccharide mutant while as the positive control showed the presence in excessively high amount (Fig. 4). The data for the carbohydrate shown over here are not only contributed by the exopolysaccharides but part of free sugars can also be added up. But this amount should be very low. 


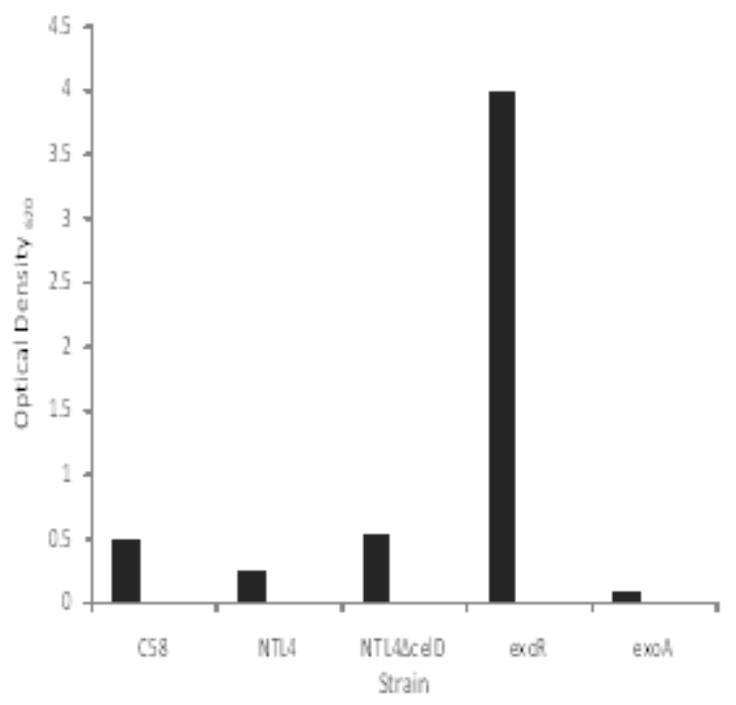

Fig. 4. Estimation of exopolysaccharide of reducing sugar by anthrone

The involvement of cellulose in bacterial biofilms has long been known for Acetobacter xylinum, which forms a biofilm at air-water interfaces and on the surface of decaying fruit (Cannon \& Anderson 1991). More recently, cellulose has been observed to be involved in biofilm formation by Escherichia coli, Salmonella enteritidis and S. typhimurium (White et al. 2003, Zogaj et al. 2001). P. fluorescens forms a biofilm containing acetylated cellulose (Spiers et al. 2003). Previous studies have shown that the absence of cellulose reduces that ability of $A$. tumerfaciens to bind to plant surfaces and to colonize roots and severely attenuates virulence (Matthysse 1983, Minnemeyer et al. 1991). Mutants of P. fluorescens and $S$. enteriditis that are unable to make cellulose also fail to form a biofilm (Spiers et al. 2003, White et al. 2003).

In this study the biofilm formed on the abiotic surface by $A$. tumefaciens wildtype was compared with that formed by the cellulose deficient mutant. The cellulose deficient mutant NTL4A celCD showed slight increased biofilm formation in PVC coverslips compared to NTL4 strain when grown in static culture. The NTL4 strain differs from the mutant strain in the cellulose biosynthesis gene only. The cellulose mutant strain has deletion in the celC and celD region of the cellulose biosynthesis gene. The wild type C58 however showed excessive biofilm formation.
Thus the results from biofilm assay demonstrated that in spite of abolishing the cellulose synthesis property by truncating the cellulose synthesis gene, the biofilm formation has not been affected.

This might indicate that cellulose is not an obligatory compound for biofilm formation. Since various exopolysaccharides are secreted by bacteria contributing to biofilm formation, polysaccharide other than cellulose might be playing important role in biofilm formation.

The results from Calcofluor plate assay and anthrone assay illustrated the presence of extracellular polysaccharide in cellulose mutant strain of $A$. tumefaciens. In fact, the slight increase in biofilm formation and extracellular polysaccharide in NTL4A cel CD compared to NTL4 strain that is not deficient in cellulose biosynthesis must be related with gene for polysaccharide other than cellulose might have been better expressed in absence of cellulose gene

The major component for the biofilm formation is still unknown since several different compounds are associated with the formation of biofilm. There are several exopolysaccharides released by bacteria known to facilitate in the formation of biofilm. The $A$. tumefaciens genome encodes for production of at least six polysaccharide species, several of which play roles in attachment and biofilm formation. These include the UPP adhesin, cellulose, succinoglycan, cyclic â1,2-glucans, $\beta$-1,3-glucan (curdlan), and outer membrane lipopolysaccharide (LPS) (Heindl et al. 2014). Though synthesis of cellulose fibrils have been associated with root colonization of $A$. tumefaciens (Matthysse 1983), it is still unknown if cellulose has important role in the biofilm formation in an inert material.

Outer membrane protein OmpA of $E$. coli decreased cellulose production which increased biofilm formation on polystyrene surface (Ma \& Wood 2009). Increasing cellulose production by overproducing AdrA was found to decrease biofilm formation on hydrophobic PS plates by Ma \& Wood (2009). Several factors can be associated with biofilm formation. Cellulose was found to decrease biofilm formation in curli-mediated biofilm formation on PP microtitre plates (Gualdi et al. 2008). Curli help adherence of $E$. coli to intestinal epithelial cells and coexpression of 
Reshma Tuladhar/Biofilm formation of Agrobacterium.......

curli and cellulose decreases the adherence (Wang et al. 2006).

Though production of cellulose enhances attachment to plant root (Matthysse et al. 2005) the mechanism of binding on PVC surface may not be identical. Cellulose being hydrophilic in nature the attachment might be enhanced on hydrophilic plant root surface but reduced in hydrophobic PVC. Identifying the factors that reduce the biofilm formation in inanimate object will have implication in overcoming the problems of biofilm formation in medical devices such as catheters.

\section{Acknowledgments}

The author is thankful to Prof. Dr. Clay Fuqua and members of Fuqua Lab, Indiana University, Bloomington, IN, USA.

\section{References}

Binns, A.N., R.D. Joerger and Jr J.E. Ward. 1992 Encyclopedia of Microbiology. Vol 1, Academic Press, pp 37-51.

Bogino, P.C., L. Oliva Mde, F.G. Sorroche and W. Giordano. 2013. The role of bacterial biofilms and surface components in plant-bacterial associations. Int.J. Mol. Sci. 14:15838-15859.

Cangelosi, G.A., L. Hung, V. Puvanesarajah, G. Stacey, D.A. Ozga, J.A. Leigh and E.W. Nester. 1987. Common loci for Agrobacterium tumefaciens and Rhizobium meliloti exopolysaccharide synthesis and their roles in plant interactions. J.Bacteriol. 169:2086-2091.

Cannon, R.E. and S.M. Anderson. 1991. Biogenesis of bacterial cellulose. Crit. Rev. Microbiol. 17:435-447.

Flemming, H.C. and J. Wingender. 2010. The biofilm matrix. Nat. Rev. Microbiol. 8:623-633.

Gelvin, S.B. 2003. Agrobacterium-mediated plant transformation: the biology behind the "genejockeying” tool. Microbiol. Mol. Biol. Rev. 67:16-37.

Gualdi, L., L. Tagllabue, S. Bertagnoli, T. Lerano, C. De Castro and P. Landini. 2008. Cellulose modulates biofilm formation by counteracting curli-mediated colonization of solid surfaces in Escherichia coli. Microbiology. 154:2017-2024.

Heindl, J.E., Y. Wang, B.C. Heckel, B. Mohari, N. Feirer and C. Fuqua. 2014. Mechanisms and regulation of surface interactions and biofilm formation in Agrobacterium. Front. Plant Sci. 5(176):1-21.

Ma, Q. and T.K. Wood. 2009. OmpA influences Escherichia coli biofilm formation by repressing cellulose production through the CpxRA two-component system. Environmental Microbiol.11(10): 2735-2746.

Mathee, K., O. Ciofu, C. Sternberg, P.W. Lindaum, J.I. Campbell, P. Jensen, A.H. Johnsen, M. Givskov, D.E. Ohman, S. Molin, N. Hoiby and A. Kharazmi. 1999. Mucoid conversion of Pseudomonas aeruginosa by hydrogen peroxide: a mechanism for virulence activation in the cystic fibrosis lung. Microbiol. 145:1349-1357.

Matthysse, A.G. 1983. Role of bacterial cellulose fibrils in Agrobacterium tumefaciens infection. J. Bacteriol. 154:906-915.

Matthysse, A.G. 1986. Initial interactions of Agrobacterium tumefaciens with plant host cells. Crit. Rev. Microbiol. 13:281-307.

Matthysse, A.G., M. Marry, L. Krall, M. Kaye, B.E. Ramey, C. Fuqua and A.R. White. 2005. The effect of cellulose overproduction on binding and biofilm formation on roots by Agrobacterium tumefaciens. Molecular PlantMicrobe Interactions. 18:1002-1010.

Minnemeyer, S.L., R. Lightfoot and A.G. Matthysse. 1991. A semi-quantitative bioassay for relative virulence of Agrobacterium tumefaciens strains on Bryophyllum daigremontiana. J. Bacteriol. 173:7723-7724.

Parsek, M.R. and C. Fuqua. 2004. Biofilms 2003: emerging themes and challenges in studies of surface-associated microbial life. J. Bacteriol. 186:4427-4440.

Reuhs, B.L., J.S. Kim and A.G. Matthysse AG.1997. Attachment of Agrobacterium tumefaciens to carrot cells and Arabidopsis wound sites is correlated with the presence of a cell-associated, acidic polysaccharide. J. Bacteriol. 179(17):5372-5379.

Spiers, A.J., J. Bohannon, S.M. Gehrig and P.B. Rainey. 2003. Biofilm formation at the air-liquid interface by the Pseudomonas fluorescens SBW25 wrinkly spreader requires an acetylated form of cellulose. Mol. Microbiol. 50:15-27.

Sutherland, I. 2001. Biofilm exopolysaccharides: a strong and sticky framework. Microbiology. 147:3-9.

Wang, X., M. Rochon, A. Lamprokostopoulou, H. Lünsdorf, M. Nimtz and U. Römling. 2006. Impact of biofilm matrix components on interaction of commensal Escherichia coli with the gastrointestinal cell line HT29. Cell Mol. Life Sci. 63:2352-2363.

White, A.P., D.L. Gibson, S.K. Collinson, P.A. Banser and W.W. Kay. 2003. Extracellular polysaccharides associated with thin aggregative fimbriae of Salmonella enteric serovar enteritidis. J. Bacteriol. 185:5398-5407.

Zogaj, X., M. Nimtz, M. Rohde, W. Bokranz and U. Romling. 2001. The multicellular morphotypes of Salmonella typhimurium and Escherichia coli produce cellulose as the second component of the extracellular matrix. Mol. Microbiol. 39:1452-1463. 
Nepal Journal of Science and Technology Vol. 15, No.2 (2014) 41-46 